\title{
The Low Frequency Radiation and Scattering of Sound from Bubbly Mixtures Near the Sea Surface
}

\author{
William M. Carey ${ }^{\circ}$ and Ronald A. Roy ${ }^{\dagger}$ \\ 'Dept. of Ocean Engineering, MIT, 77 Massachusetts Ave, Cambridge, Ma. 02139 \\ ${ }^{\dagger}$ Dept. of Aerospace and Mechanical Engineering, Boston University, 110 Cummington St., Boston, Ma 02215
}

\begin{abstract}
The radiation and scattering from micro-bubble clouds with volume fractions greater than $.01 \%$ are described with a theoretical model and measured data. In the low frequency limit the radiation and scattering of sound was determined to be monopole in the free field. The natural frequency of the cloud is predicted by a modified Minnaert equation. The measured back scatter target strength was found to be consistent with theoretical expectations.
\end{abstract}

\section{BACKGROUND}

Micro-bubble plumes/clouds are produced when waves break and are convected to depth. The fundamental question is: What role is played by these micro-bubble plumes in the production of sound and the scattering of sound near the sea surface from the low $(20 \mathrm{~Hz})$ to mid $(2 \mathrm{kHz})$ frequency range? Measurements of ocean ambient noise show a dramatic increase in mid-frequency ambient noise levels when wave breaking occurs. Furthermore measurements of the sound scattering from the sea surface has a large zero-Doppler component. If micro-bubble clouds and plumes with void fractions, vfs, greater than .01\% act as collective resonant oscillators, then radiated noise can be produced and scattering can occur with little Doppler shift but ample spread. This hypothesis is based on the theory that when a large number of micro-bubbles with individual resonance frequencies far above the frequency of excitation are present in an acoustically compact region, the mixture properties determine the radiation and scattering. In such a mixture, acoustic propagation can be described by an effective wave equation with mixture density and sound speed determined by vf, $\chi$, and the following equation:

$$
\mathrm{C}_{\mathrm{mlf}}^{2}=\mathrm{P} / \rho_{\ell} \chi(1-\chi), \quad 0.002<\chi<0.94
$$

At low frequencies $(<1 \mathrm{kHz})$ it is the volume fraction, not the bubble-sizes, that matters most. The solution for sound scattering from a compliant sphere in a fluid can be found in texts. The cloud has no well defined boundary but is localized by perhaps by subsurface vorticity. It is acoustically compact with radius $r_{0}$, composed of bubbles with resonance frequencies greater than the excitation frequency, and undergoes global rapid compression. The radiation from such a compact region is described by a multipole expansion. The monopole resonance frequency is

$$
f_{o} \approx \frac{1}{2 \pi r_{0}}\left(\frac{3 y P}{\rho_{1} \chi}\right)^{1 / 2}
$$

a modified Minnaert formula. For a gas bubble $\chi=1$ and $\gamma$ is the adiabatic constant; however for the LF radiation from a cloud $\gamma=1$, an isothermal process. Equation 2 follows because $f_{o}^{2}$ is proportional to the compressibility of the bubbly region, characterized by it's stiffness $\left(4 \pi r_{o} \rho C_{m \theta t}^{2}\right)$ and inertia $\left(4 \pi r_{o}^{3} \rho / 3\right)$. For the case of air, $C_{a} \approx 340 \mathrm{~m} / \mathrm{s}$ and $\rho_{a} / \rho=0.0013$; we find the resonance frequency of a gas bubble of radius $a$ to be $f_{o b}=3.379 / a(H z)$, and for the cloud, $\chi=0.002$ and $C_{b v f}=200 \mathrm{~m} / \mathrm{s}$, and the resonance frequency is $f_{o x} \approx 55 / r_{o}(\mathrm{~Hz})$. If the equilibrium size of each gas bubble is $50 \mu \mathrm{m}$, then we have $f_{a b}=67.6 \mathrm{kHz}$, and if the radius of the air cloud is $r_{o}=0.25 \mathrm{~m}$, we find that $f_{o c} \approx 220 \mathrm{~Hz}$. Thus, groups of micro-bubbles with radii between $10-50 \mathrm{~cm}$ (compared to a $1.5 \mathrm{~cm}$ radius air bubble) can have resonance frequencies in our range of interest, $-220 \mathrm{~Hz}$. This analysis shows that micro-bubble clouds with vfs greater than $0.01 \%$ can be treated as compact sources of sound.

\section{THE TIPPING TROUGH EXPERIMENT}

To test this radiation hypothesis, a precisely actuated tipping trough was used to produce bubble clouds for the 
far field acoustic tests conducted in a deep lake and pond. The trough, $0.305 \mathrm{~m}$ above the water surface, emptied into an acoustically transparent, butyl-rubber salt-water containment bag. A video microscope was used to determine the temporal evolution of the bubble-size distribution. In these experiments, all bubbles had resonance frequencies greater than $0.66 \mathrm{kHz}$ with peak temporal vfs between 16 and $20 \%$ with mean vfs $\approx 5 \%$. Each tip produced a transient sequence of sounds from the initial water entry followed by the evolution of the bubbly mixture. Far-field spectra showed a peak at $100 \mathrm{~Hz}$; since $3.4 \mathrm{~cm}$-radius bubbles were not observed, the radiation originated from the mixture. The measurements were repeatable with initial potential energy of 123-169 J, sound energy flux of $0.4-2.510^{-5} \mathrm{~J}$ (energy flux source levels of $95-104 \mathrm{~dB} \mathrm{re} \mu \mathrm{Pa}^{2}$ sec. @ $1 \mathrm{~m}$ ), and radiation efficiencies of $0.3-2.2610^{-3}$. Calculations (eqn. 2) using the measured bubble cloud volume and vfs yielded frequencies $\approx 100 \mathrm{~Hz}$.

\section{THE SCATTERING EXPERIMENT}

Parametric (PS) and conventional sources (CS) with conventional receivers were used to measure the back scatter of a rising bubble cloud. Although the PS has the advantage of LF directionality, bubbly liquids are highly nonlinear. The contribution of enhanced parametric interaction within the cloud was eliminated by comparison with CS results. Bubble clouds were produced at $91.4 \mathrm{~m}$ depth using a pressurized steel enclosure with a concentric circular array of 4822 -gauge hypodermic needles. A 3.5 - $\mathrm{sec}$ burst of air at $68.95 \mathrm{kPa}$ overpressure produced a cylindrical cloud with a length $\approx 1.7 \pm 0.3 \mathrm{~m}$, radius $\approx 0.24 \pm 0.01 \mathrm{~m}$, with a vf $\approx 0.4 \pm 0.007 \%$. Bubble radii were normally distributed about $1.1 \pm 0.2 \mathrm{~mm}$, corresponding to $f_{o} \approx 9.2 \pm 1.7 \mathrm{kHz}$.The target strengths were high $(\approx-.31 \mathrm{~dB})$ at the resonance frequency of $325 \mathrm{~Hz}$. The monopole resonance frequency calculated with eqn. 2 using the cloud volume was $324 \pm 53 \mathrm{~Hz}$ in agreement with measurements. The TS is given by eqn. 3 where $I_{S}$ and $I_{i}$ are the scattered and incident intensities. When interference from the bubbler was included, the calculated TS of $3.9 \pm 0.8 \mathrm{~dB}$ was comparable to the measured TS of $-3.4 \pm .9 \mathrm{~dB}$; excellent agreement.

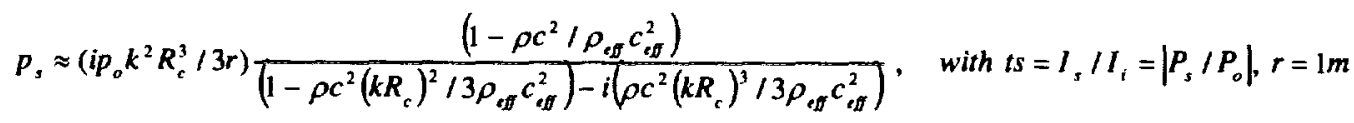

\section{CLOUDS NEAR THE SEA SURFACE}

The aforementioned experiments confirm the treatment of a complex cloud at low frequencies behaves as a monopole. For a cloud a distance $d$ beneath a plane surface characterized by a reflectivity coefficient $\mu$, $-1 \leq \mu \leq 0$; the method of images yields this expression for the near-surface, backscatter target strength:

$$
T S_{n s}=T S_{f f}+10 \log \left\{+\mu^{2}+2 \mu-4 \mu \sin ^{2}\left(k d \sin \theta_{s}\right)\right\}^{2},
$$

where $\theta_{s}$ is the grazing angle measured relative to the horizontal. The reflection coefficient $\mu=-1$ corresponds to a smooth pressure release surface and $\mu=0$ corresponds to an extremely rough surface. For $k d<<1$ and $\mu=-1$, the scattered pressure scales with $(d / \lambda)^{*}$. Although high vf clouds possess significant cross sections at resonance, they are also subject to substantial image interference owing to their close proximity to the surface. A cloud resonant at $100 \mathrm{~Hz}\left(r_{o s} \approx 0.12\right.$ to $0.28 \mathrm{~m}$ and $v f, \chi, \approx .2 \%$ to $\left.1 \%\right)$ with a free-field TS $=0$ to $8 \mathrm{~dB}$ placed $0.5 \mathrm{~m}$ under the surface possesses a $\operatorname{Ts}_{n s}\left(10^{\circ} \leq \theta_{8} \leq 15^{\circ}\right)=-31$ to $-46 \mathrm{~dB}$. Thus, this cloud would appear as a point-like return over the experimental and Chapman-Harris values between -50 to $-55 \mathrm{~dB}$ at $15-\mathrm{knot}$ wind speed.

\section{CONCLUSIONS}

Bubble clouds from breaking waves can radiate and scatter sound near the sea surface. During the first few seconds of the dynamic cloud evolution, sound radiates whenever a portion of the cloud meets the resonant conditions and is forced by the dynamics of compression. The radiation frequency is described by a Minnaert formula for the individual bubbles and for groups of bubbles within the cloud when modified by the vf. These clouds can also scatter sound over a longer period of time provided the vf is sufficient. The observed effect would appear on a sonar image as "point-like" returns on a more diffuse background. 